\title{
Hepatic Lobectomy-Case Report of Two Patients, Performed at Chittagong Medical College Hospital
}

\author{
SMA ALI ${ }^{\mathrm{a}}$, MM HUSSAIN ${ }^{\mathrm{b}}$
}

\begin{abstract}
Summary:
Lobectomy of liver is indicated for different conditions; mainly malignancy and trauma. Though it is highly specialized operation and preferably be performed in specialized tertiary center, sometime a general surgeon may not be in a position to avoid the surgery due to very low socioeconomic condition or to tackle emergency traumatic condition. Two case report of Lobectomy of liver; right lobectomy for hepatocellular carcinoma, and
\end{abstract}

Introduction:

Hepatic resection for tumor removal was first performed more than a century $\mathrm{ago}^{1}$. Liver resection is indicated to a wide range of pathological conditions of liver. These include: (i) benign primary tumours such as liver cell adenoma or haemangioma; (ii) primary malignant tumours such as hepatocellular carcinoma; (iii) hepatic trauma; or (iv) extensive hepatolithiasis limited to on anatomical lobe. The most frequent indication, however, is liver metastases, the commonest being colorectal metastases. ${ }^{2}$.

With the understanding of liver segmental anatomy described by Tung and most importantly by Couinaud resection rate has been increased dramatically during last couple of years. Due to different condition including continuing increase of incidence of trauma, a general surgeon cannot avoid liver resection to save life in some compelling situation. More over operations on the liver and pancreas have fallen within the domain of the general surgeon and have been part of general surgery training ${ }^{3}$.

Application of recent technological support and ground-breaking techniques of resection has evolved

a. Dr. SM Ashraf Ali, FCPS, Associate Professor of Surgery, Chittagong Medical College

b. Dr. Md. Margub Hussain, FCPS, MHPEd, Professor of Surgery, Dhaka Medical College.

Address of correspondence: Dr. SM Ashraf Ali, FCPS, Associate Professor of Surgery, Chittagong Medical College. Email: ashrafa@bttb.net.bd

Received: 21 July, 2007

Accepted: 5 August, 2008 left lobectomy for left lobe big cyst and multiple stones in it were performed at Chittagong Medical College Hospital utilizing the existing facilities. Both patients recovered uneventfully. Case I expired after two month and fourteen day, Case II leading a pain free cheerful normal life.

Experience of management of these two cases of resection of liver is recorded at Chittagong Medical College Hospital.

(J Bangladesh Coll Phys Surg 2008; 26: 153-157)

aiming to reduce blood loss, morbidity and mortalities related to hepatic resection ${ }^{4,5}$.

\section{Case-1}

Mr. Mostafiz 35 year's male admitted in to medicine unit on 03/08/2003 with epigastric pain and vomiting for one day without significant past illness. The case was labeled as one of acute abdomen with probable diagnosis of acute pancreatitis or perforation of peptic ulcer.

Next morning he was transferred to surgical unit. Examination of the patient revealed severe pallor, pulse rate $120 / \mathrm{m}$, Blood pressure $80 / 40 \mathrm{~mm} \mathrm{Hg}$ with diffuse rigidity and tenderness involving whole abdomen. Plain X-ray abdomen showed diffuse opacity without evidence of free gas and serum amylase was normal. The patient was resuscitated. But condition of the patient was not improved significantly. We decided to explore but patient's condition did not allow anaesthesia. A drain tube was inserted in to the peritoneal cavity under local anesthesia to drain collection. Almost fresh blood came out. Post operatively the patient improved and become stable within few days.

We decided to investigate further. Ultrasonography of the hepatobiliary system showed -liver was normal in size. A doubtful rounded area- approximately $3.5 \mathrm{~cm}$ $\mathrm{x} 3.0 \mathrm{~cm}$ was detected in the posterior aspect of the right lobe of the liver just below the diaphragm. CT scan not done due to financial constrain. Serum alpha Feto Protein level was $300.0 \mathrm{IU} / \mathrm{ml}$. In isotop Liver Scan- there was a big photon deficient area in the antero-inferior and right lateral aspect of the right 
lobe. Rest of the right lobe and left lobe appears normal. X-ray chest, TC,DC,ESR,Hb\%, ECG, blood urea, serum creatinine, fasting and two hours after breakfast blood glucose level, liver function test ,serum albumin level, globulin level, AG ratio and serum electrolyte were normal. The patient was negative for hepatitis $\mathrm{B}, \mathrm{C}$ and $\mathrm{E}$ virus.

On 28/08/03 under general anesthesia with endotracheal intubation and muscle relaxation using roof top incision with extension to upwards and right the abdomen explored aiming to do right lobectomy. After confirmation of the pathology right lobe of the liver was mobilized by ligation and division of the ligamentum teres, division of the falciform ligament and right triangular ligament (Fig.1). After cholecystectomy right hepatic duct, right hepatic artery and right branch of the portal vein ligated and divided in between. Glisson's capsule was opened; liver tissue was separated by finger fracture technique along the line of demarcation. Strong suction was applied, and once located; vessels and bile ducts were secured, divided and ligated as dissection proceeded

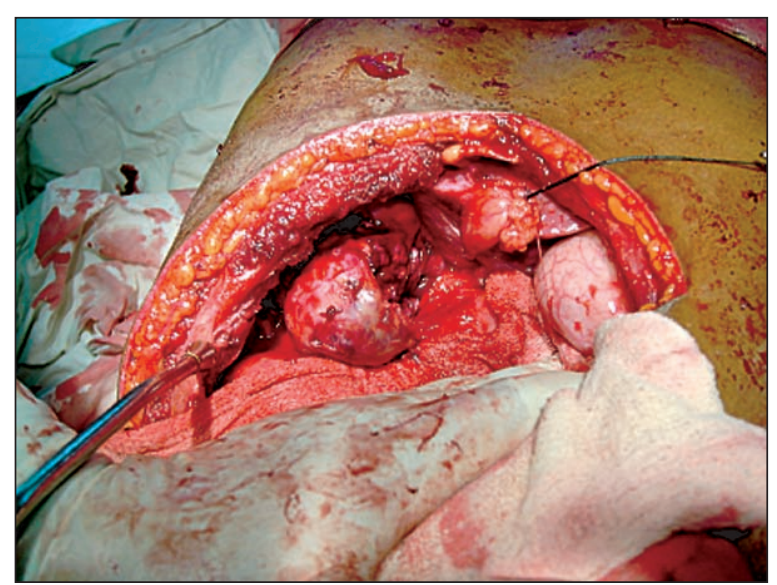

Fig.-1: Ligamentum teres is divided, falciform ligament incised \& separated. Right lobe was mobilized by incising the right triangular ligament.

(fig.2). Elevation from posterior aspect facilitated identification of major hepatic veins. These were secured within the liver substance (Fig-3). Specimen taken out, haemostasis on the cut surface was achieved by ligation and mattress suture (Fig-4). Wound closed in layers .Total duration of operation was 4 hours 15 minutes. During operation, six units of whole blood were transfused. Urine out-put was
$900 \mathrm{ml}$ (in 4 hours $15 \mathrm{~m}$ ). The patient recovered uneventfully during post operative period and oral feeding stared on $4^{\text {th }}$ postoperative day. Biochemical status and haematological profile was within normal limit except hyponatraemia $\left(\mathrm{Na}^{+} 122 \mathrm{mmol} / \mathrm{L}\right)$ and hypoalbuminemia (albumine-3g/dl) which were corrected accordingly. The patient become mildly icteric (Serum bilirubin-2.00 $\mathrm{gm} / \mathrm{dl}$ ) on $4^{\text {th }}$ postoperative day, recovered spontaneously. Serum alphafetoprotein level was $44 \mathrm{IU} / \mathrm{ml}$ on $02 / 10 / 03$.

On 28/10/03 chemotherapy with Doxorubicin and Cisplatin was started by oncologist. Patient was

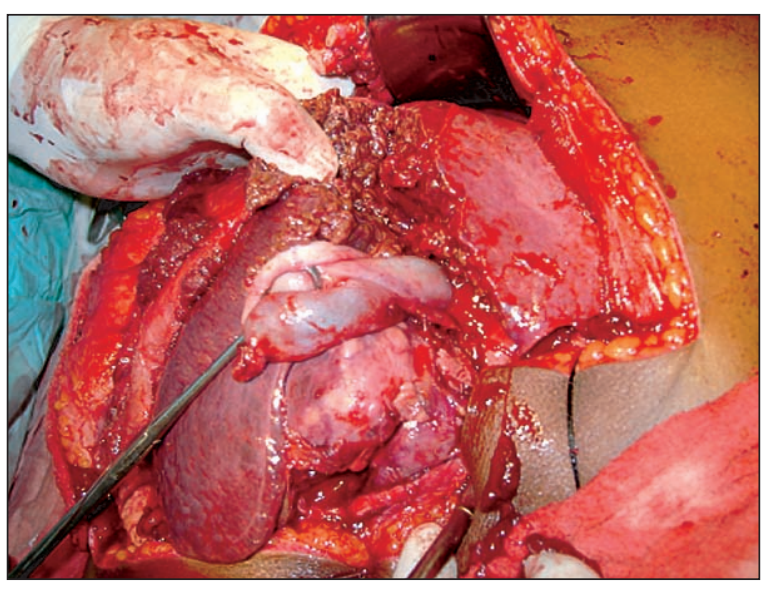

Fig.-2: Division of liver tissue and hepatic vein. Glisson's capsule opened, liver tissue is fractured. Strong suction is applied once located, vessels \&bile ducts are secured \& ligated.

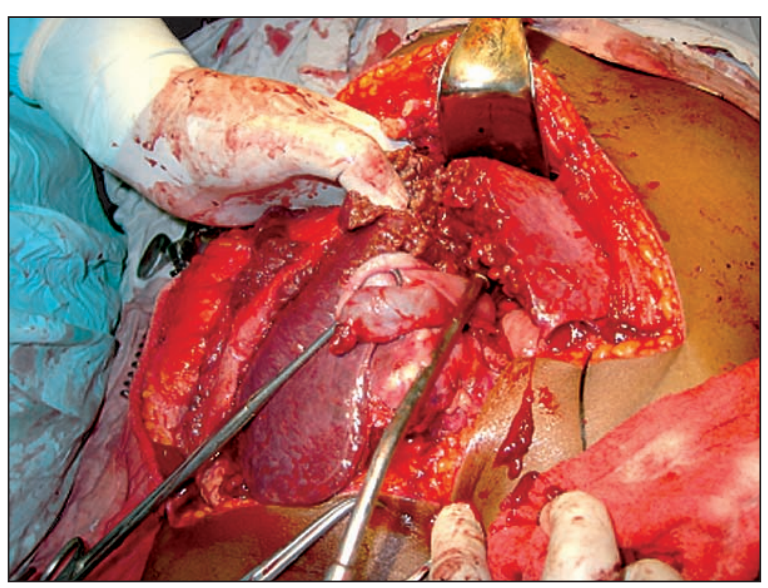

Fig.-3: Division of liver tissue continued. As the tissue is divided, the fissure is opened. Elevation from posterior aspect opens the fissure. Major hepatic veins are identified \& secured within the liver substance 


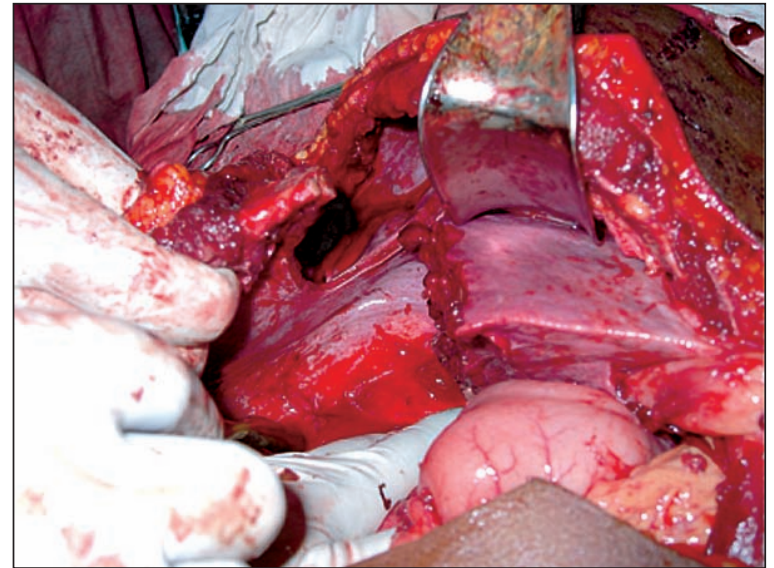

Fig.-4: Right lobe removed, haemostasis of the raw cut surface achieved by ligation and mattress suture.
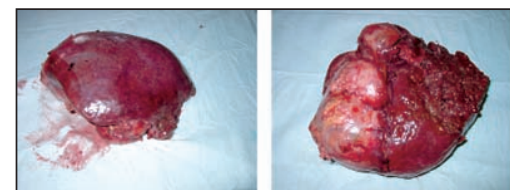

Fig.-5 a.b.: Resected specimen, showing diaphragmatic and inferion surface

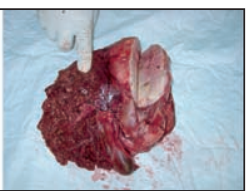

Fig.-5 c.: Resected specimen, cut surface

feeling better. Unfortunately on $09 / 11 / 03$, condition of the patient deteriorated. He developed ascites, anemia and expired on 14/11/03.

\section{Case-2}

Eighteen years old young girl referred by a physician with intermittent pain in her epigastric and left upper abdomen for 7 years with fever and vomiting. She was mildly anemic but not jaundiced. Left lobe of the liver was palpable, firm, non tender and smooth.

Ultrasonography of the hepatobiliary system revealed big irregular hypo echoic area involving the left lobe of the liver; $5.5 \times 4 \mathrm{~cm}$ in size, containing multiple hyper echoic structure with distal shadowing in it, indicating stones.

CT scan of the hepatobiliary system revealed big low attenuated area involving almost whole left lobe of the liver with multiple small hyper attenuated structure in it, indicated stones (Fig.6). Liver function test and all investigations for anesthetic fitness were within normal limit. Serologically the patient was negative for hepatitis $\mathrm{B}, \mathrm{C}$ and $\mathrm{E}$ virus.

Exploration was done through a roof top incision with a vertical extension upwards along midline (Fig.7).

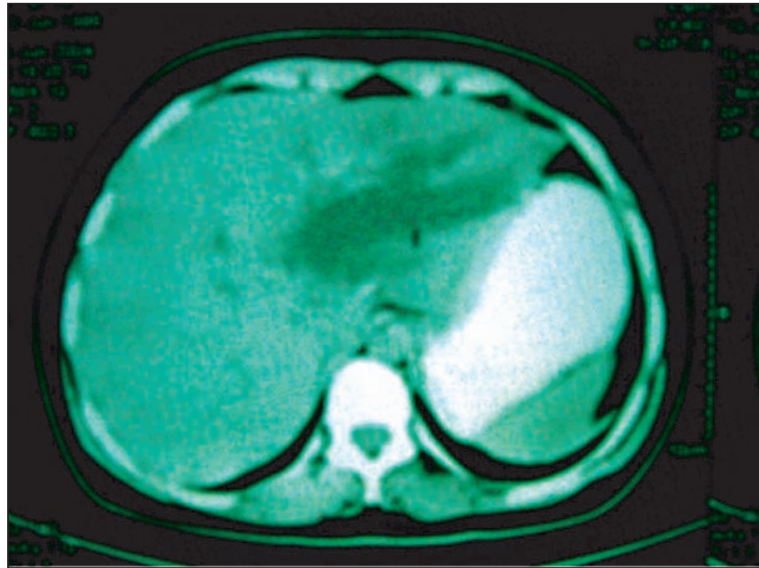

Fig.-6: CT scan of the hepatobiliary system revealed big low attenuated area involving almost whole left lobe of the liver with multiple small hyper attenuated structure in it, indicated stones.

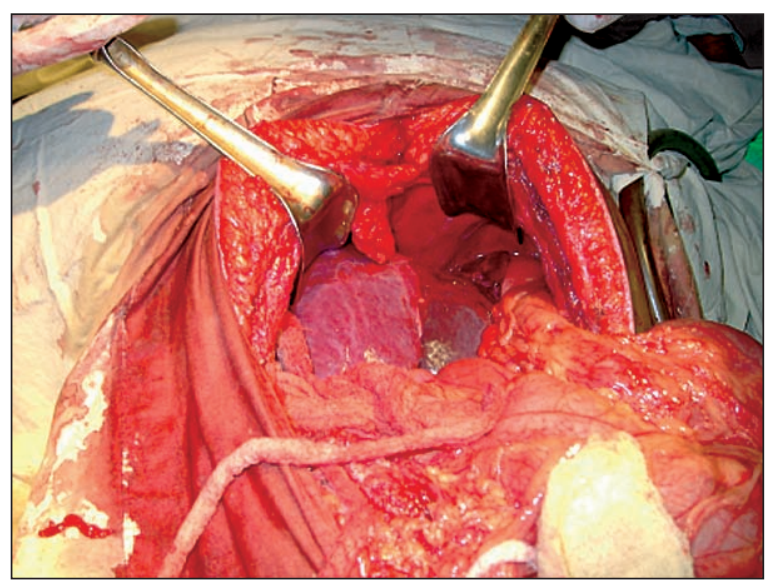

Fig.-7: Exposed left lobe of the liver.

Left lobe of the liver was mobilized by dividing left triangular ligament and proceeding medially up to inferior venacava. Left lobectomy performed following ligation and division of left hepatic duct, left hepatic artery and left branch of the portal vein at the porta hepatis . Left hepatic vein was dissected facilitated by rotation of left hepatic lobe, doubly ligated and divided. Lobectomy was then completed by finger fracture of parenchyma and securing smaller vessels and ducts (Fig.8). T tube was left in the common bile duct. Wound was closed in layers. Total duration of operation was 2 hours 50 minutes, and two units of blood were transfused during operation. Condition of the patient during operation was stable. Urine out put was $400 \mathrm{ml}$ (2 hours50m). 


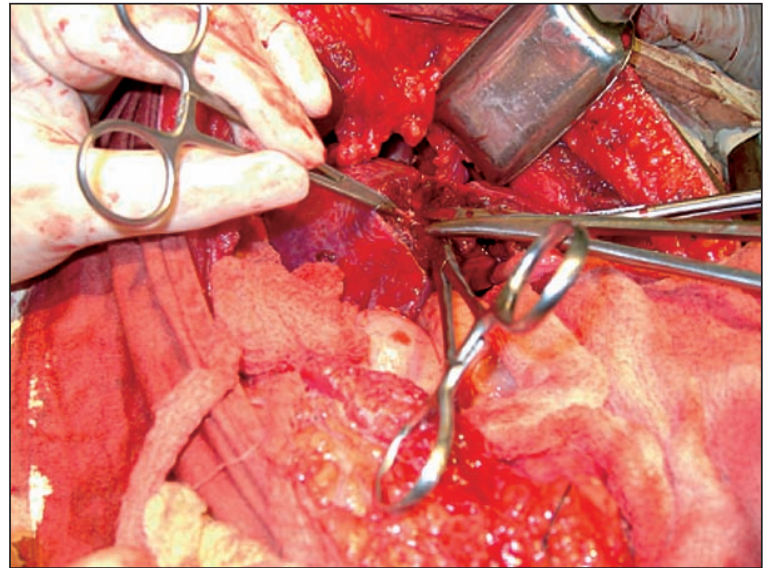

Fig.-8: Left lobectomy by finger fracture of parenchyma and securing smaller vessels and ducts.

The patient recovered uneventfully during post operative period.

T-tube cholangiogram performed on $11^{\text {th }}$ POD showed well delineated intrahepatic biliary tree in the right lobe (Fig.9). The patient was followed up for last 2 years. She is now symptom free.

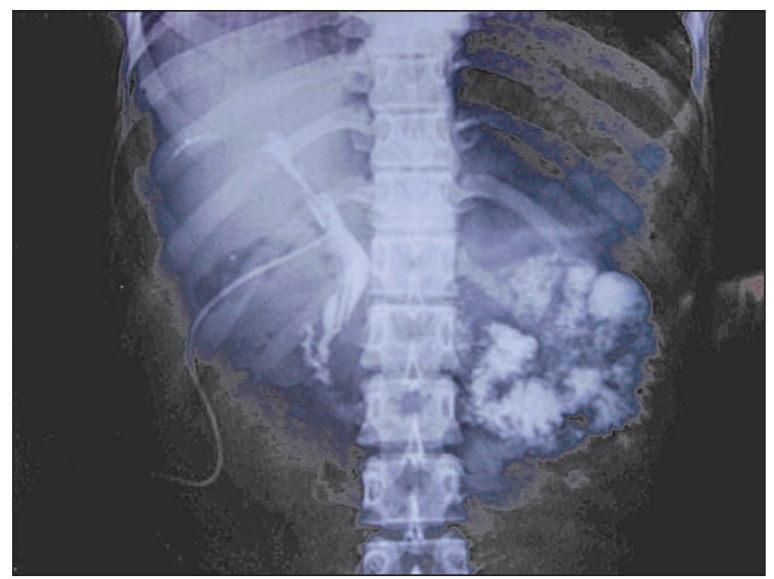

Fig.-9: Post operative T tube cholangiograme showing visualization of right hepatic duct system.

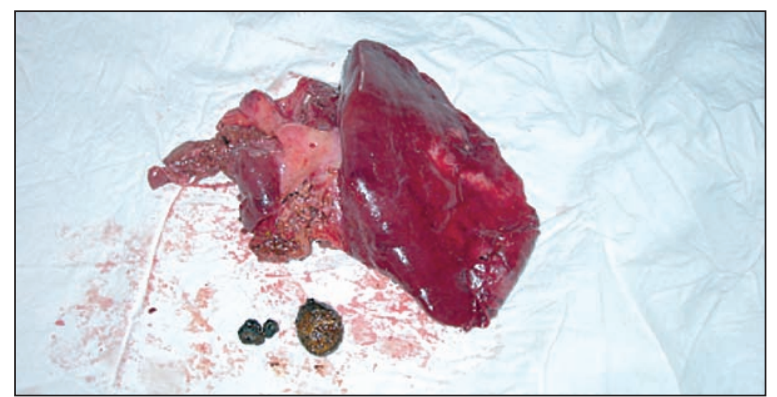

Fig.-10: Resected specimen, left lobectomy and stone.

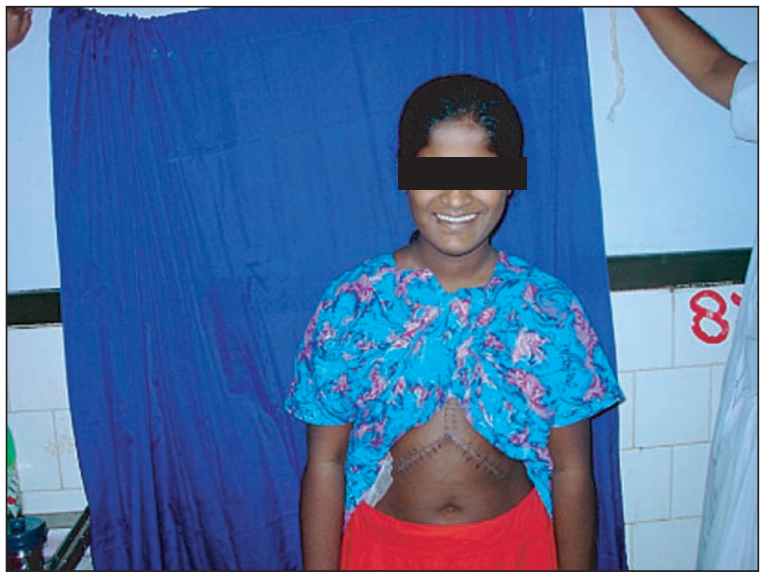

Fig.-11: Cheerful patient in the 11 th post operative day.

\section{Discussion:}

This case is seems to be the first report of successful hepatic lobectomy outside the BSMMU and BIRDEM, Dhaka. In patients with liver cancer (Hepatocellular Cancer, HCC), liver resection is limited to patients with one or two small ( $3 \mathrm{~cm}$ or less) tumors with excellent liver function. In case I, ultrasonography, serum alpha fetoprotein and isotope scan were highly suggestive of the diagnosis. Fine-needle aspiration cytology was tried but not possible due to technical difficulties. CT scan was not possible for financial constrain. The initial diagnosis of a liver tumor can be suggested by symptoms, findings on physical examination or elevation of a tumor marker, such as carcinoembryonic antigen (CEA) in a patient with a previous history of colorectal cancer, alpha-fetoprotein (AFP) in a patient with hepatocellular carcinoma ${ }^{6}$.

For HCCs less than two $\mathrm{cm}$, the detection rate by computerized tomography (CT) scanning is $72 \%$, whereas it is $93 \%$ by magnetic resonance imaging (MRI). Hepatic angiography is very useful in confirming the diagnosis of HCC because of the very particular vascular features the tumours exhibits. CT angiography/portography and delayed CT scanning after intra-arterial injection of Lipiodol are also very sensitive and specific imaging modalities to confirm the diagnosis and detect small lesions. Once HCC is suspected, the aim of further investigations is to confirm the diagnosis, stage the tumours and assess the underlying liver disease ${ }^{7}$.

After diagnosis careful preoperative evaluation is crucial to avoid unnecessary surgical explorations 
that considerably reduce the quality of the short remaining life span of patients with un resectable liver tumors. Only $20 \%$ of patients with hepatocellular carcinoma have disease that can be completely resected ${ }^{8}$.

Standard right hepatic lobectomy was done. The extent of liver resection depends on the size and location of the tumor. The relationship of the tumor to vascular structures and bile ducts determines the extent of resection. The options include: wedge, segmental, lobectomy, and trisegmentectomy. Intraoperative ultrasonography allows the accomplishment of anatomical resections otherwise not possible. Precise definition of hepatic vein anatomy and association with color Doppler enables hepatectomies otherwise not possible, expanding the indication at surgical resection ${ }^{9}$.

Surgical resection remains the mainstay of treatment in case of hepatocellular carcinoma.

The 5-year survival rate ranges from $22.5 \%$ to $51.1 \%$ depending upon methods of resection; anatomical or non-anatomical ${ }^{10}$. In the first case the acute abdominal presentation was due to hemorrhage from tumor. Very fortunately the patient survived after blood transfusion. Post operative ascites and rise of AFP could be explained by recurrence due to previous rupture of tumor mass.

In Case II, diagnosis of big cystic area containing multiple stones was made confined to left lobe. Standard left lobectomy was performed, and patient was running well. She developed a keloid and still she is under our follow-up. Standard treatment of hepatolithiasis is still controversial, ranging from conservative to liver transplantation. In a study ${ }^{11}$ of 128 patients left lateral segmentectomy compared with left hepatic lobectomy as a treatment for isolated left hepatic lobe stones. Lower rate of residual stones (4\% vs $22 \% ; \mathrm{P}<.01)$ was observed in case of left lobectomy. Percutaneous choledochoscopic removal is another modalities of treatment of hepatolithiasis. A retrospective study of 174 cases $^{12}$ revealed that success rate of hepatic resection is superior $(98 \%$ versus $70.5 \%$ ) to percutaneous choledochoscopy for hepatolithiasis.

\section{Conclusion:}

The lobectomy of liver, though highly specialized operation, can be attempted at least to tackle any emergency as well as unavoidable situation at any medical college hospital.

\section{References}

1. Tiffany LM. The removal of a solid tumor from the liver by laparotomy. Md Med J 1890; 23: 531

2. Heriot $\mathrm{AG}$, Karanjia ND. A review of techniques for liver resection. Ann R Coll Surg Engl 2002; 84: 371-380.

3. Helling TS, Khandelwal A. The Challenges of Resident Training in Complex Hepatic, Pancreatic, and Biliary Procedures. J Gastrointest Surg. 2007; 23: 310-314.

4. Rees M, Plant G, Wells J, Bygrave S. One hundred and fifty hepatic resections: evolution of technique towards bloodless surgery. Br J Surg.1996; 83: 1526-9.

5. Hanna SS, Nam R, Leonhardt C. Liver resection by ultrasonic dissection and intraoperative ultrasonography. HPB Surg. 1996; 9: 121-8.

6. Armando S, Alisher A, Gail C. Management of Primary and Metastatic Tumors to the Liver. Oncology. 1996; 10(6): $911-$ 930.

7. Usatoff V, Habib N. Hepatic malignancy: challenges and opportunities for the surgeon. J.R.Coll.Surg.Edinb. 2000; 45, April: 99-109.

8. Junginger T, Kneist W, Dünschede F, Von L C, Seifert J, Kanzler S. Liver metastases of colorectal carcinoma-how often can be operated upon?. Zentralbl Chir. 2007; Aug; 132(4): 281-6 (Abstract).

9. Torzilli G, Leoni P, Gendarini A, Calliada F, Olivari N, Makuuchi M. Ultrasound-guided liver resections for hepatocellular carcinoma. Hepatogastroenterology. 2002; Jan-Feb; 49(43): 21-7.

10. Ziparo V, Balducci G, Lucandri G, Mercantini P, Di Giacomo G, Fernandes E. Indications and results of resection for hepatocellular carcinoma. Eur J Surg Oncol. 2002; Nov; 28(7): 723-8.

11. Sun WB, Han BL, Cai JL. The surgical treatment of isolated left-sided hepatolithiasis: a 22-year experience. Surgery 127(5): 493-7, May 2000.

12. Moon TC, Philip CH. Liver Resection for Intrahepatic Stones. Arch Surg. 2005; 140: 993-997. 